\title{
Challenges and Strategic Research Plans for Earth and Heliosphere: Research Infrastructures, Projects and Initiatives
}

\author{
Ingrid Mann ${ }^{1}$, Kirsti Kauristi ${ }^{2}$, Ruth Bamford ${ }^{3}$, Ian McCrea ${ }^{3}$, Joeran \\ Moen $^{4}$, Kjellmar Oksavik ${ }^{5}$, Masanori Yamauchi ${ }^{6}$, Magnar Johnsen \\ Gullikstad $^{7}$, Esa Turunen ${ }^{8}$ \\ ${ }^{1}$ UiT, The Arctic University of Norway, Tromsoe, Norway \\ ${ }^{2}$ Finnish Meteorological Institute, Helsinki, Finland \\ ${ }^{3}$ Rutherford Appleton Laboratories, United Kingdom \\ ${ }^{4}$ University of Oslo, Norway \\ ${ }^{5}$ University of Bergen, Norway \\ ${ }^{6}$ Swedish Institute of Space Physics, Kiruna, Sweden \\ ${ }^{7}$ Troms Geophysical Observatory, Tromsoe, Norway \\ ${ }^{8}$ Sodankylae Geophysical Observatory, Finland
}

\begin{abstract}
We describe existing research infrastructures relevant for space weather and open issues of space weather research including the need for sustainable observation networks and for high-quality data products as basis for model development. The local relevance in Europe for studies of the ionosphere at high latitude is described. We propose as possible a way forward to sustain space weather research in Europe to establish a European research infrastructure project for space weather research and observations.
\end{abstract}

Keywords. Heliospheric physics, ionospheric variability, space weather

\section{Introduction}

The region around the Sun that is filled with the solar wind, the heliosphere includes almost the entire solar system. It is influenced by plasma, particles and radiation disturbances launched by the Sun, and by the physical processes that they cause. Research to study of these phenomena started in the 1970's, when observations from space provided observational data of the solar corona and the interplanetary medium inside Earth orbit and the first space missions to the outer system were launched. As a result, the International Astronomical Union (IAU) devoted a new commission to the topic of heliospheric studies (described in a recent summary of the commissions history, Mann et al. 2016). The first report of the new IAU commission pointed out that, in future, it would be useful to develop a capability for predicting, from solar observations, the state of the solar wind at the Earth (cf. Axford et al. 1976). The report also noted already that such a predictive capability would need to be based on advanced theoretical understanding. While some researchers realized very early the influence of space processes on Earth and sometimes used the term weather in this context, the term space weather is frequently used since the 1990s (cf. Cade and Chan-Park 2015). Space weather (SW) describes the immediate impact on the Earth of the time-varying cosmic environment; this cosmic environment is influenced predominantly, but not exclusively by plasma, particles, magnetic field, and radiation launched by the Sun, the disturbances and the physical processes that they cause. Hence SW is linked to a number of different areas of basic research including areas of interest represented within the IAU. Today we have a wealth of data, but the need 
to integrate and understand them in many ways poses a greater challenge than ever: How can we coordinate our efforts, make results accessible and sustain relevant observation initiatives? This article will address some of the open issues, it provides a short description of the top-down international efforts, local space weather challenges, existing infrastructures and the plans for a Research Infrastructure for Space Weather Research in Europe. The IAU can play an important role of providing a forum of bottom-up science discussions and initiatives related to space weather research.

\section{Space Weather: International top-down coordination}

During recent years much progress has been made in using the data from missions and activities to provide, develop and maintain services for Space Weather (SW) environment specification, now-casting and short-term forecasting. To improve international coordination, both in science and in operational services, COSPAR, the international Committee on Space Research, has addressed this challenge together with NASAs ILWS (International Living With a Star) programme, by publishing a Roadmap (Schrijver et al. 2015), which gives a review of the science questions that need to be solved in order to answer the questions posed by future forecast services. It is recognized that there is a need for reliable forecasts with $<1$ day lead times and more accurate information about the onset time, duration, amplitude and spatial distribution of SW events than can be provided today.

One of the key recommendations made by the Roadmap is the development of innovative approaches for the incorporation of data into models (including data-driving, assimilation and ensemble modelling). For example, longer lead times in the forecasting of Interplanetary Magnetic Fields (IMF) north-south component, Bz would need better understanding of how a CME evolves as it leaves the Sun and propagates through the heliosphere. Similarly, resolving the key factors controlling different energy dissipation modes in the disturbed magnetosphere-ionosphere system would enable more accurate specification of the near-Earth radiation environment, particle precipitation and electric fields, informing strategies to mitigate harmful effects due to Geo-magnetically Induced Currents (GICs) in power transmission lines. This kind of prediction cannot reliably be performed at present, because our current capabilities in predicting ionospheric storm evolution are still moderate, due to the limited understanding of some crucial magnetosphere-ionosphere-thermosphere linkages and consequent deficiencies in the physics-based models. In order to improve the current situation in modelling and forecasting, the COSPAR Roadmap makes recommendations both for enhanced teaming inside the solar-terrestrial research community, and involving collaborations between agencies and with other communities (e.g. meteorology, solid-earth). These recommendations are of particular interest for European research groups who typically push forward their SW projects with sporadic funding, compiled from different national and international sources.

\section{International research infrastructures and global networks}

There are already a number of international efforts to integrate and coordinate the activities that are relevant to space weather. Within Europe, the participants in ESAs SSA space weather programme develop a coordinated set of Expert Service Centres in the areas of solar weather, heliospheric weather, ionospheric weather, space radiation and geomagnetic conditions. The results from these are mediated through a Space Weather 
Coordination Centre, which coordinates the provision of space weather services and operates a helpdesk, dealing with queries about the precursor service provision or space weather conditions in general. An ESA SWE data centre hosts a space weather web portal, in which are embedded a number of space weather applications, provided by the Expert Service Centres. The ESA SSA programme is also working toward the development of underpinning space-based operational capabilities, notably the Lagrange missions to the L1 and L5 points, being pursued in collaboration with NASA, which will ensure the availability continuous monitoring data, from both upstream of the Earth, and from a location well away from the Sun-Earth line, allowing both in-situ observation and remote sensing of potentially Earth-impacting space weather events. All of this is already a major step forward, compared to the situation a few years ago; however there are still two areas of major weakness which ESA funding is unable to address. These relate to the sustainability of the ground-based instrument networks which underpin the provision of space weather data and the $\mathrm{R} 2 \mathrm{O}$ development of the modelling capabilities which use these data in order to produce simulations and predictions.

The ground-based instruments are very diverse in nature, ranging from relatively small and cheap detectors operated by individual research groups to large, complex instruments which are already international research infrastructures in their own right. The EISCAT Scientific Association, for example, has effectively functioned as an international research infrastructure since 1975, providing a network of incoherent scatter radars in the European Arctic, with funding support from relevant research organisations in Norway, Sweden, Finland, UK, Japan and China; in earlier times the EISCAT funders also included France and Germany. Since 1998, EISCAT has been working within the framework of ESFRI and EU funding to plan its new EISCAT_3D radars, three large phased arrays (cf. Wannberg et al. 2010), to be situated in the northern parts of Norway, Sweden and Finland. This facility, which is about to enter its construction phase, is capable of continuous operation with modes which can be adapted as geophysical conditions change. A focus of the project lies in studies related to ionospheric processes and to the long-term variability in the atmosphere and global change (cf. McCrea et al. 2015). As well as investigating fundamental physical processes, a significant part of the case for the new radar has been based on its ability to provide continuous data for the emerging set of space weather monitoring and forecast capabilities. It should also be noted in passing that EICSCAT_3D has capabilities for the surveillance and tracking of space debris, whose dynamics are linked to variations in space weather and potentially point to a significant future user base for space weather services.

To study global phenomena also requires a world-wide coordination of observations. The EISCAT radars already run a number of experiments per year which are jointly coordinated with similar radars in Canada, the US, Puerto Rico, Peru and Greenland, with occasional participation by radars in Russia, Ukraine, China and Japan, planned on an international level but sustained on a best efforts basis according to the available funding. Another notable international initiative in radar collaboration is SuperDARN, a collaboration of nine countries, which has been continuously running a network of HF oblique sounding radars in the high-latitude regions to study plasma convection and other phenomena for more than 20 years (Greenwald et al. 1995) and has recently added poleward (PolarDARN) and equatorward (StormDARN) extensions of the instrument network. An example for a wide-spanning network established on national level is the Chinese meridian project that involves a chain of ground-based observatories roughly along 120 degree $\mathrm{E}$ longitude and 30 degree $\mathrm{N}$ latitude. that are equipped with multiple instruments to measure key parameters of the ionosphere at 20 to $1000 \mathrm{~km}$ (Wang 2014). 


\section{Local infrastructures}

At the other end of the spectrum, small instruments, such as magnetometers, are operated by individual research groups around the world, but their data are coordinated together by collaborations such as SuperMAG, which provides a central repository for over 300 globally distributed instruments in a standard form, with common standards of data reduction and artefact removal. Similar examples of coordinated use of multiple distributed instruments can be seen in the MIT Automated Process of GPS (MAPGPS) system, which integrates data from over 2000 globally distributed GPS receivers, and the GLORIA riometer consortium, which provides a database of instruments measuring ionospheric absorption worldwide and offers some degree of data synthesis (e.g. to create multi-instrument movies). Even at European level, these networks tend to be operated on a best efforts basis, sometimes by a single funder and occasionally by a collection of national funding agencies acting together. Successful examples are often operated by one or more national funding agencies to operate a set of instruments locally that are integrated in an international structure. For example, the Finnish Meteorological Institute leads the MIRACLE network of magnetometers, all-sky cameras and GPS measurements, distributed across Norway, Sweden and Finland, the magnetometer data from which also form part of the SuperMAG database, while the GPS data are also used in global assimilative studies. In general terms, however, the present arrangements for funding space weather instrument operations in Europe are far from satisfactory, threatening the stability of any system based on exploiting their data.

\section{Model development and validation}

The development of space weather models has, to a large extent, always been a pure research activity in which groups with appropriate capabilities have developed models as research tools to be applied to understanding particular physical problems. This has meant that the design, documentation and validation of such models has always varied greatly. In particular, within Europe at least, such models have tended to remain as

EISCAT VHF radar 6 September 2017 Observation - quick-look data
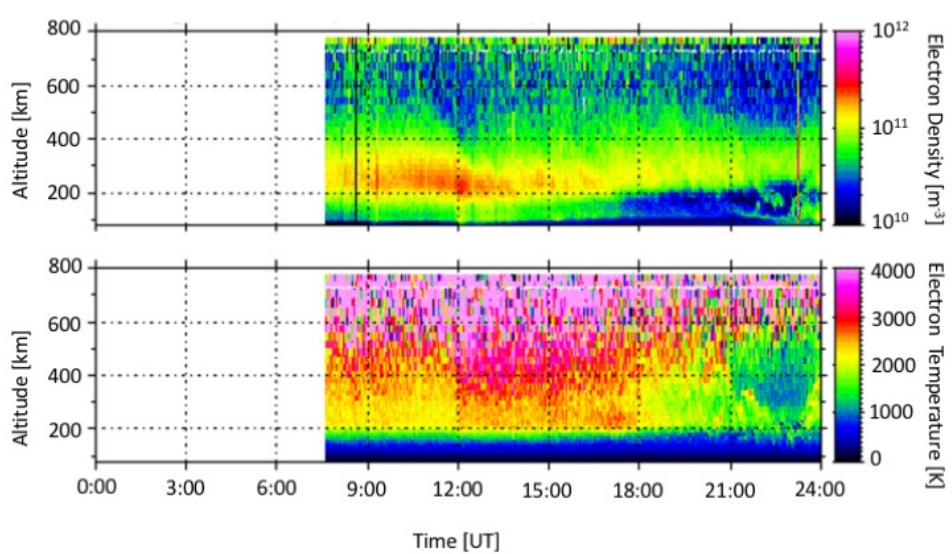

Figure 1.*

Figure 1: The ionospheric electron density and temperature measured with EISCAT on 6. September 2017 showing a clear signature in electron temperature at about 200 to $600 \mathrm{~km}$ altitude following the X9.3 solar flare around 12:00 UT. 
research tools, where the understanding and ability to extend them for particular purposes, or to use them to their full potential, has been restricted to a relatively small group of developers. There are several examples of models which have become obsolete as developers have moved on and such knowledge has been lost. Up to now there has been no good mechanism for models to be translated from research to operational use, though this is beginning to change as both ESA and national space weather centres are realising the requirement for operational models. In the US, the job of upgrading models for operational use has been taken on, at least in part, by NASAs Community Coordinated Modelling Center (CCMC), which works both with developers and operational users to bridge this gap, turning research models into operational tools. This method has been very successful, because it has ensured developer involvement in the operational use of their models and also ensured that the intellectual property of developer groups is acknowledged and respected. Nothing similar currently exists in Europe and establishing a proper mechanism for model development in Europe would be important and timely.

\section{Local space weather challenges}

Some of the space weather challenges have a particular local relevance. Europe includes populated regions in the Arctic and with the intensification of human activity in the Polar Regions relying on satellite signals, a detailed understanding of the different mechanisms structuring the ionosphere at high latitudes is of increasing practical importance (cf. Moen et al. 2013). Ionospheric irregularities are known to degrade the signal quality of the Global Navigation Satellite System (GNSS), and this may reduce GNSS position accuracy (Jin et al. 2015). Intense GNSS phase scintillation and loss of signal lock has been found in the dayside polar cap (Oksavik et al. 2015). In the central polar cap the scintillation is often localized to high-density storm-time tongues of ionization or polar cap patches that are in transit. The strongest scintillation events are found in the nightside polar cap, where dense polar cap patches enter the night-side auroral oval. As the irregularities can vary both in time and space, a 3D multi-scale instrument approach is required to understand underlying mechanisms, instability modes, and growth rates causing the plasma turbulence. Characterizing the evolution of ionospheric irregularities and the transfer of energy between the different scales can be regarded as a study of fundamental plasma physics from a theoretical point of view, but it also has direct applications to developing reliable space weather forecast.

\section{European initiative for a space weather research infrastructure}

The need to establish a sustainable setting for regular and coordinated transition of research models to operational space weather services has triggered some informal discussions among European research groups. The Research-To-Operation (R2O) projects conducted under the Space Situational Awareness (SSA) programme of ESA are acknowledged, but on the other hand it is noticed that some research groups possessing valuable capabilities may not have easy access to the SSA programme. Therefore, it would be advisable to have a research oriented framework (with working title European Sustainable SW Network, ESWNET) to facilitate efficient networking between SW modelers and observers, based initially on the distributed network of experts in existing models and data products as the substrate for developing and validating new models. ESWNET would exploit EU investments for e-infrastructures facilitating advanced computation environments and the network would work closely with academic research groups and 
other stakeholders (e.g. ESA and EUMETSAT) who maintain ground- and space-based instrumentation.

Figure 2 presents a schematic, showing how ESWNET could be located within the European environment of SW activities. The new network would facilitate communication and coordination between ESA SSA SW services and national and EU research programmes, with the goal to integrate the best expertise from distributed sources into one joint Sun-Earth observatory. ESWNET could support SSA by serving as the platform for upgrading Technical Readiness Levels and validation of new SW models and measurement concepts. In an optimal situation, this network could even influence the planning of European and national SW funding programs, so that SW service user requirements could be addressed with coordinated research missions.

Obviously ESWNET itself would also need some funding in order to be an attractive platform for research groups to collaborate. Getting a position in the Roadmap of the European Strategy Forum on Research Infrastructures (ESFRI) could be a feasible pathway for establishing ESWNET. The ESFRI framework, which operates with the backing of the European Commission, provides good models for agreeing on governance structures, data policy and IPR issues. Furthermore, as partners in an ESFRI initiative, ESWNET research groups would have a stronger position in the competition for sustainable national funding lines.

\section{Summary}

The discipline of space weather is becoming an area in which scientific understanding needs to inform the requirements for real-time monitoring and future prediction. This need, which will only become more urgent as society becomes increasingly dependent on space-based technologies, can only be fulfilled by using the capabilities of our globally distributed observing instruments, complemented by the best of our models, optimised to work together in a real-time operational setting. While some organisations, notably ESA in Europe and NOAA and NASA in the US, are taking steps toward this, some

\section{European Sustainable Space Weather Network}

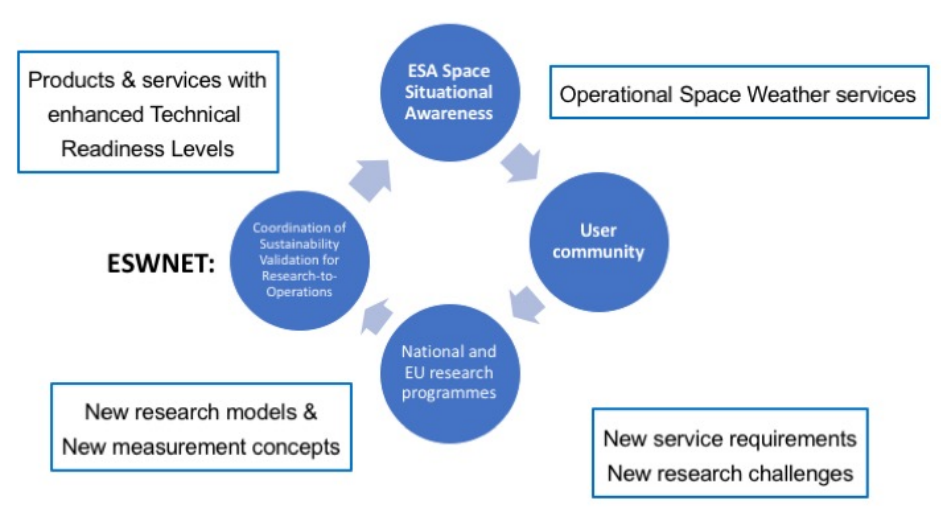

Figure 2. *

Figure 2. A new network of European solar-terrestrial research groups, ESWNET, is suggested to support ESA and EU in coordinating both operational and research activities for continuously improving Space Weather services. 
key capabilities are still missing. In particular, there is a worrying lack of stability and sustainability in the funding arrangements for the operation of ground-based instruments, which is notable in Europe but also applies on a global scale. Another missing piece in the jigsaw is the effort, and governance framework, needed to turn research tools such as models into real operational systems, capable of using appropriately validated multi-instrument data to make predictions with appropriately quantified accuracy. These responsibilities would seem to fall somewhere between the research community, which uses instrument data and develops models to study fundamental physical mechanisms, and operational agencies which have a requirement to use data and models in a standard way for operations. A new European Space Weather Infrastructure, as proposed here, would supply the missing pieces in this jigsaw, and deliver a stable European contribution to this burgeoning international collaborative effort.

\section{Acknowledgements:}

The quick-look data presented in Figure 1 are courtesy of EISCAT and for illustration purpose only. EISCAT is an international association supported by research organizations in Norway (NFR), Sweden (VR), Finland (SA), Japan (NIPR and STEL), China (CRIPR), and the United Kingdom (NERC). EISCAT VHF and UHF data are available under http://www.eiscat.se/madrigal/. KK and IM acknowledge discussions on SWNET with Jean Lilensten, Consuelo Cid Tortuero, Iwona Stanislawska, Jens Berdermann, Johan de Keyser, Margit Haberreiter, Peter Gallagher, Stefaan Poedts, Vladimir Kalegaev, and Ari Viljanen. KO is grateful for being selected as the 2017-2018 Fulbright Arctic Chair, and his sabbatical at Virginia Tech is sponsored by the U.S.-Norway Fulbright Foundation for Educational Exchange. KO is also supported by the Research Council of Norway under contract 223252 .

\section{References}

Axford, W. I., Hollweg, J. V., Suess, S. T., Blum, P. W., \& Fahr, H. J. 1976, Trans. Int. Astron. Union (Reports on Astronomy) 16A, 175-187

Greenwald, R.A. et al.\} 1995, Darn/Superdarn, Space Sci. Rev. 71, 761-796

Jin, Y., Moen, J.I., \& Miloch, W. J. 2015, J. Geophys. Res. Space Physics 120, 91769191, doi:10.1002/2015JA021449

Mann, I., Manoharan, P.K., Gopalswamy, N., Briand, Chashei, I. V., Gibson, S.E., Lario,D. Hanaoka, Y., Malandraki,O., Kontar, E., \& Richardson, J.D. 2016, Division E Commission 49, Transactions of the IAU 29A, 300-315

McCrea, I, et al. 2015, Prog. Earth Planet. Sci. 2, 21, doi:10.1186/s40645-015-0051-8

Moen, J., Oksavik, K., Alfonsi, L., Daabakk, Y., Romano V., \& Spogli, L. 2013, J. Space Weather Space Clim. 3, A02, doi:10.1051/swsc/201302

Oksavik, K., van der Meeren, C., Lorentzen, D. A., Baddeley, L. J., \& J. Moen, J. I. 2015, J. Geophys. Res. Space Physics 120, 91619175, doi:10.1002/2015JA021528

Schrijver, C.J., Kauristie, K., et al. 2015, Advances in Space Research 55, 2745-2807 